\title{
PARTICLE-PARTICLE CORRELATIONS AND THE SPACE-TIME STRUCTURE OF HEAVY ION COLLISIONS
}

\author{
Wolfgang BAUER \\ National Superconducting Cyclotron Laboratory \\ and Department of Physics, Michigan State University \\ East Lansing, MI 48824-1321, USA \\ Electronic mail address: BAUER@MSUNSCL
}

\begin{abstract}
The present status of the use of two-particle intensity interferometry as a diagnostic tool to study the space-time dynamics of intermediate energy heavy ion collisions is examined. Calculations for the two-proton and two-pion correlation functions are presented and compared to experiment. The calculations are based on the nuclear Boltzmann-Uehling-Uhlenbeck transport theory.
\end{abstract}

\section{KEYWORDS}

Heavy ion transport theory; two-particle interferometry; two-pion correlation function; two-proton correlation function; pion shadowing; nuclear equation of state; in-medium nucleon-nucleon cross sections.

\section{INTRODUCTION}

Probably the premier goal in performing heavy ion collisions at intermediate and high beam energies is to obtain information on the nuclear matter phase diagram, on how nuclear matter behaves under compression and heating, and on possible phase transitions in nuclear matter. Trivially, the time development of the process with which we hope to compress and heat nuclei, the collision of two heavy ions, is not directly observable to us. To obtain this information we are exclusively dependent on the particles emitted during the course of the heavy ion reaction. Some of these particles are created in the reaction process (e.g. photons, pions, kaons, etas, anti-proton, ...). Other particles 
have already been present in the initial stages of the reaction (protons and neutrons) and only have their momenta and energies modified during the collision.

All of these particles are observed a long time after the reaction has taken place. We are only able to observe the final four-momenta of these particles, but not the time development that led to these final states. Furthermore, we are not able to directly measure the location from which these particles were emitted. Thus we are confronted with a scenario in which we can only observe information which is integrated over space and time. It is our task to unfold this information to reconstruct the history of the heavy ion reaction and to possibly extract the physically relevant parameters of the nuclear equation of state.

In this sense heavy ion reaction physics is like forensic medicine, in which minute tell-tale signs and pieces of evidence left behind are collected in order to reconstruct a crime.

The last decade has seen an extensive investigation of inclusive single particle spectra from heavy ion collisions. The basic idea (Stock et al., 1982) is that compression of nuclear matter requires energy, which is then not available for the production of secondary particles. By first comparing to intranuclear cascade simulations (Stock et al., 1982) and then to nuclear transport calculations including mean field effects (Bertsch et al., 1984; Aichelin and Ko, 1985) it first seemed that there was a clear signal for the value of the compressibility of nuclear matter. However, further studies including momentum dependent nuclear mean field potentials (Aichelin et al., 1985; Gale et al., 1987; Gale, 1987), medium effects on the elementary scattering process (Cugnon et al., 1987), and improved elementary hadron-hadron cross sections (Li and Bauer, 1991, 1991a; Danielewicz and Bertsch, 1991) have shown that the connection between nuclear compressibility and produced particle spectra is not as striking as previously thought. The present status of the theory of particle production (Cassing et al., 1990) indicates that there is at most a factor of 2 difference in yield of produced particles if one changes the nuclear compressibility constant, $\kappa$, between values of 200 and $380 \mathrm{MeV}$. This small sensitivity to the nuclear compressibility is due to the fact that the amount of compressional energy stored during the heavy ion collisions is roughly the same for all values of $\kappa$. Instead of storing more energy in compression, nuclei rather adjust the maximum density reached in heavy ion collisions.

The basic problem with examining single particle inclusive cross sections is again due to the fact that we are only observing a time and space integrated quantity. This problem is somewhat less severe for high energy photons, because they are produced very early in the reaction (Bauer et al., 1986) and have very little final state interaction. But pion and to some extend even kaon spectra are strongly modified by final state interactions. In addition, at beam energies of around $1 \mathrm{GeV} \mathrm{A}$, the production process of these particles can in some cases be dominated by collisions of nuclear resonances in the nuclear medium, i.e. by processes for which we do not know the elementary hadron-hadron cross sections from experimental data. Thus inclusive particle production cross sections carry only limited information on the space-time development of heavy ion reactions.

One can then ask if two-particle correlations carry more information on the space-time history of the heavy ion reaction process. The motivation for this is that by observing single particles in the final state one integrates the dynamical information over their entire world line, whereas by using two-particle correlations one has two independent world lines, the history of which one integrates over. Since the relative wave function of the two particles is modified due to their interaction at the point at which the world lines cross or come close enough (if they do), two-particle correlations can serve as a possible source of information on the space time development of heavy ion reactions.

In this paper I will focus on the utilization of two-particle correlations at small relative momenta, at 
which intensity interferometry is possible.

Intensity interferometry was introduced by Hanbury Brown and Twiss (1954, 1956, 1956a) as a technique for astronomical distance measurement. They recorded the two-photon correlation function for incoming coincident photons as a function of their relative momentum. This correlation function can be written as:

$$
R\left(\vec{k}_{1}, \vec{k}_{2}\right)=\frac{\left\langle n_{12}\right\rangle}{\left\langle n_{1}\right\rangle\left\langle n_{2}\right\rangle}-1,
$$

where $\left\langle n_{12}\right\rangle$ is the probability of detecting two coincident photons of wavenumber $\vec{k}_{1}$ and $\vec{k}_{2}$ in detectors 1 and 2 , and $\left\langle n_{i}\right\rangle$ is the probability of detecting a photon of momentum $\vec{k}_{i}$ in detector $i$ $(i=1,2)$. Equation 11 contains only count rates, which are proportional to the absolute squares of the amplitudes. As a consequence, HBT interferometry is insensitive to phase shifts introduced by atmospheric disturbances. It can be used with very large base lines and delivers superior resolution. This was first shown (Hanbury Brown and Twiss, 1956a) by measuring the angular diameter of Sirius.

The physical basis of the HBT effect is that two photons have a non-zero correlation function due to the symmetrization of their wave functions, a consequence of the quantum statistics for identical particles.

A similar technique can also be used for source size determinations in subatomic physics. This was first utilized by Goldhaber et al.(1960) in studying angular distributions of pions in $p \bar{p}$ annihilation processes. They found that the emission probability of coincident identical pions is strongly affected by their Bose-Einstein statistics, which causes an enhancement of the correlation function at zero relative momentum, $q=0$. The width of the maximum at $q=0$ depends on the radius of the interaction volume (Goldhaber et al., 1960) and also on the life-time of the emitting source (Shuryak, 1973).

In recent years, two-pion intensity interferometry has been strongly pursued at ultra-relativistic energies, where the interest is on the interplay between source dynamics and final state interaction (Pratt, 1984; Kohlemainen and Gyulassy, 1986) and pion correlations from an exploding source (Pratt, 1986). Intensity interferometry derives its main attraction, however, from the prospect of its possible use as a diagnostic tool for the formation of quark-gluon plasma (Pratt, 1986; Bertsch et al., 1988).

Intensity interferometry is not restricted to bosons, but can also be applied to Fermions. Koonin (1977) proposed to use two-proton intensity interferometry to obtain 'pictures' of heavy ion collisions. The advantage of using protons as a probe lies in the fact that they are already present in the colliding nuclei and can be liberated relatively easily. In contrast, to create a pair of pions one has to spend an energy $E_{\min }=2 m_{\pi} \approx 280 \mathrm{MeV}$ in the center of mass of the generating system. Therefore, protons can be used as a probe at much lower energies. In addition, the two-proton relative wave function contains the prominent ${ }^{2} \mathrm{He}$-'resonance', which leads to enhanced sensitivity of the correlation function to the source size. Finally, protons are easy to detect with the required resolution.

Recent progress has been centered around the theoretical computation of two-proton correlation functions from nuclear transport theory (Gong et al., 1990, 1991, 1991a; Zhu et al., 1991, Bauer et al., 1992, 1992a, 1992b). In this framework, it is possible to understand the dependence of the correlation functions on the spatial dimension of the emitting source, on the momentum distribution of particles in the source, and on the time development of the system emitting the particles. Comparisons of this theory to experimental data have established two-proton intensity interferometry as a quantitative tool to study heavy ion reaction dynamics. 
A summary of the present status of the field as well as further references can be found in Boal et al. (1990) and Bauer et al. (1992). Here I primarily focus on the use of two proton and two pion intensity interferometry as a diagnostic tool for reconstruction of the space-time history of intermediate energy heavy ion reactions and with it the determination of limits on the parameters of interactions of nucleons in dense nuclear matter.

\section{HEAVY ION TRANSPORT THEORY FOR INTERMEDIATE ENERGIES}

During the last few years, several groups have developed a nuclear transport theory for intermediate energy $\left(20 \mathrm{MeV} \leq E_{\text {beam }} / A \leq 2 \mathrm{GeV}\right.$ ) heavy ion reactions (Bertsch et al., 1984; Kruse et al., 1985, 1985a; Grégoire et al., 1985, 1987; Bauer et al., 1986; Li and Bauer, 1991; Danielewicz and Bertsch, 1991). This transport theory describes the time evolution of the nuclear one-body Wigner distribution $f(\vec{r}, \vec{p}, t)$ under the influence of the nuclear mean field and individual nucleon-nucleon collisions via the Boltzmann-Uehling-Uhlenbeck (BUU) equation

$$
\begin{aligned}
\frac{\partial}{\partial t} f(\vec{r}, \vec{p}, t)+ & \frac{\vec{p}}{m} \vec{\nabla}_{r} f(\vec{r}, \vec{p}, t)-\vec{\nabla}_{r} U \vec{\nabla}_{p} f(\vec{r}, \vec{p}, t) \\
= & \frac{g}{2 \pi^{3} m^{2}} \int d^{3} q_{1^{\prime}} d^{3} q_{2} d^{3} q_{2^{\prime}} \\
& \delta\left(\frac{1}{2 m}\left(p^{2}+q_{2}^{2}-q_{1^{\prime}}^{2}-q_{2^{\prime}}^{2}\right)\right) \cdot \delta^{3}\left(\vec{p}+\vec{q}_{2}-\vec{q}_{1^{\prime}}-\vec{q}_{2^{\prime}}\right) \cdot \frac{d \sigma}{d \Omega} \\
& \cdot\left\{f\left(\vec{r}, \vec{q}_{1^{\prime}}, t\right) f\left(\vec{r}, \vec{q}_{2^{\prime}}, t\right)(1-f(\vec{r}, \vec{p}, t))\left(1-f\left(\vec{r}, \vec{q}_{2}, t\right)\right)\right. \\
& \left.\quad-f(\vec{r}, \vec{p}, t) f\left(\vec{r}, \vec{q}_{2}, t\right)\left(1-f\left(\vec{r}, \vec{q}_{1^{\prime}}, t\right)\right)\left(1-f\left(\vec{r}, \vec{q}_{2^{\prime}}, t\right)\right)\right\} .
\end{aligned}
$$

Here $U$ is the mean field potential, which is commonly parametrized as a density functional

$$
U(\rho(\vec{r}))=\alpha\left(\frac{\rho(\vec{r})}{\rho_{0}}\right)+\beta\left(\frac{\rho(\vec{r})}{\rho_{0}}\right)^{\sigma},
$$

where the constants $\alpha, \beta$, and $\sigma$ are determined from the choice of a nuclear compressibility, $\kappa$, and from the conditions $E / A=-15.75 \mathrm{MeV}$ and $d(E / A) / d \rho=0$ at $\rho=\rho_{0}$. Popular choices are (Bertsch et al., 1984) $\sigma=7 / 6 \Rightarrow \alpha=-358.7 \mathrm{MeV}, \beta=304.6 \mathrm{MeV}$ ('soft', $\kappa=200 \mathrm{MeV}$ ), $\sigma=2 \Rightarrow \alpha=$ $-124.4 \mathrm{MeV}, \beta=70.3 \mathrm{MeV}$ ('stiff', $\kappa=380 \mathrm{MeV}$ ), or (Bauer et al., 1986) $\sigma=4 / 3 \Rightarrow \alpha=-218.1$ $\mathrm{MeV}, \beta=164.0 \mathrm{MeV}$ ('medium', $\kappa=235 \mathrm{MeV}$ ). $d \sigma / d \Omega$ is the energy dependent in-medium nucleonnucleon scattering cross section, which is taken from free space elementary cross section data where available, and from detailed balance and isospin symmetry arguments otherwise.

All present solution schemes for the above equation employ the test particle method (Wong, 1982), in which the entire phase space is divided into small cells, whose equations of motion are first order differential equations in time,

$$
\begin{aligned}
\frac{d}{d t} \vec{p}_{i} & =-\vec{\nabla} U\left(\vec{r}_{i}\right)+\sum_{j \neq i} \frac{q_{i} q_{j}}{\left(\vec{r}_{i}-\vec{r}_{j}\right)^{2}}+\mathcal{C}\left(\vec{p}_{i}\right) \\
\frac{d}{d t} \vec{r}_{i} & =\frac{\vec{p}_{i}}{\sqrt{m_{i}^{2}+p_{i}^{2}}}, \\
i & =1, \ldots,\left(A_{t}+A_{p}\right) \mathcal{N}
\end{aligned}
$$

where $A_{t}$ and $A_{p}$ are the target and projectile masses, respectively, and $\mathcal{N}$ is the number of test particles per nucleon (usually taken $>100$ to reduce artificially generated numerical fluctuations). 
Fig. 1: Time evolution of the coordinate space density $\rho(x, 0, z)$ in the reaction plane (in units of $\rho_{0}$ ) for the reaction ${ }^{93} \mathrm{Nb}+{ }^{93} \mathrm{Nb}$ at a beam energy per nucleon of $60 \mathrm{MeV}$ and 0 impact parameter as calculated from the solution of equations 1 and 5 . 
The term $\mathcal{C}\left(\vec{p}_{i}\right)$ represents the solution of the collision integral via an intranuclear cascade (Cugnon et al., 1981, 1982; Cugnon and Lemaire, 1988) for the test particles. The test particle collisions respect the Pauli exclusion principle due to the presence of the factors $(1-f)$, which are numerically implemented via a Monte Carlo rejection method. In our particular numerical realization the values of $f(\vec{r}, \vec{p}, t)$ are stored in a six-dimensional lattice so that the computation of the factors $(1-f(\vec{r}, \vec{p}, t))$ only requires the call of $2^{6}$ lattice elements for a six-dimensional interpolation (Bauer, 1988).

Further details of the numerics and physics of heavy ion transport theory can be obtained from the review articles of Stöcker and Greiner (1986), Bertsch and Das Gupta (1988), Schuck et al. (1989), Cassing and Mosel (1990), and Wang et al. (1991).

The solution of equations 4 and 5 provide us with a time evolution of the single particle phase space density $f(\vec{r}, \vec{p}, t)$ and thus yield (within the model and on a semi-classical level) complete information on the heavy ion reaction history. At present this is the best and probably only valid way to visualize the nucleus-nucleus collision process. By comparing the predictions of the model to experimental data one can iteratively check the approximations entering the simulations and refine them.

For illustration we display in fig. 1 the time evolution of the reaction ${ }^{93} \mathrm{Nb}+{ }^{93} \mathrm{Nb}$ at a beam energy per nucleon of $60 \mathrm{MeV}$ and 0 impact parameter. Displayed is the nucleon density (in units of $\rho_{0}$ in the reaction plane $(y=0)$. For this simulation (Bauer et al., 1992c) a number of $\mathcal{N}=1000$ test particles per nucleon was used. The beam direction is along the $\mathrm{z}$-axis. We can see the two nuclei touching $(t=10 \mathrm{fm} / \mathrm{c})$ and interpenetrating and compressing each other $(t=40 \mathrm{fm} / \mathrm{c})$, an expansion phase $(t=70-100 \mathrm{fm} / \mathrm{c})$, and finally the formation of a ring-shaped remnant.

It should be noted in this context, however, that the model does not contain the formation of complex intermediate mass fragments other than those resulting from the decay of projectile and target remnants. A large number of groups presently work on the inclusion of fluctuations into the dynamics in order to overcome this difficulty (Bauer et al., 1987; Ayik and Grégoire, 1988, 1990; Randrup and Remaud, 1990; Burgio et al., 1991, 1992; Bonasera et al., 1992, 1992a; Randrup, 1992; Reinhard and Suraud, 1992; Ayik et al., 1992; Benhassine et al., 1992). The verdict on the success of these attempts to describe nuclear fragmentation is still out, and it may well be that a true Nbody quantum theory is needed. Present attempts to approximate this theory include transport models with dynamical production of $A \leq 3$ fragments (Danielewicz and Bertsch, 1991; Danielewicz and Pan, 1992) and on so-called 'Quantum Molecular Dynamics', semiclassical N-body simulations (Aichelin and Stöcker, 1986; Aichelin, 1991; Ono et al., 1992; Peilert et al., 1992).

\section{SINGLE PARTICLE PROTON SPECTRA}

Since the solution of Equation 2 represents the time evolution of the single particle distribution function $f(\vec{r}, \vec{p}, t)$, it is possible (in the limit of $t \rightarrow \infty$ ) to predict all single particle observables such as proton spectra (Aichelin and Bertsch, 1986; Bauer, 1987a) in this theory.

As one example we show in fig. 2 the comparison of theoretical calculations and experimental data for the single particle inclusive proton production cross sections. The calculations are represented by the histograms and are the results of the calculation of 40000 different events at random impact parameters. The data (Fox et al., 1986; Chitwood et al., 1986) are represented by the circles. As one can see, the overall normalization as well as the energy and angle dependence of the experimental data are well reproduced. There is a maximum disagreement of about a factor of 2 between calculation 
Fig. 2: Inclusive single proton energy spectra for the reactions ${ }^{16} \mathrm{O}+{ }^{12} \mathrm{C}$ at $\mathrm{E} / \mathrm{A}=25 \mathrm{MeV}$ and ${ }^{12} \mathrm{O}+{ }^{12} \mathrm{C}$ at $\mathrm{E} / \mathrm{A}=40 \mathrm{MeV}$. The calculations (Bauer, 1987a) are represented by histograms and represent the result of the simulation of 40000 reactions at randomly distributed impact parameters. The data (Fox et al., 1986; Chitwood et al., 1986) are represented by the circles.

and measurement. Thus we can be confident that the phase space distribution function integrated over coordinate space, $\int d^{3} r f(\vec{r}, \vec{p}, t)$, reproduces the experimental observables in the limit $t \rightarrow \infty$.

\section{LARGE-ANGLE CORRELATIONS BETWEEN PROTONS}

Taking the calculated single-particle distributions $f(\vec{r}, \vec{p}, t)$, we cannot make predictions for twoparticle correlations. However, if these correlations are simply consequences of the conservation laws for momentum, energy, angular momentum, and particle number, then it is possible to obtain limited information on the two-particle correlation function, if one calculates in the ensemble method. It was shown (Bauer, 1987a) that two-proton correlation functions measured at large angles can be successfully reproduced by the BUU theory, provided that total momentum conservation is correctly taken into account. In another investigation Ardouin et al. (1988) found that the variation of the large angle correlation function with polar angle $\theta$ is largely due to angular momentum effects. 


\section{CALCULATION OF CORRELATIONS AT SMALL RELATIVE MOMENTUM}

Even though it is impossible to produce two-particle correlation functions by only taking the calculated single particle distribution functions $f(\vec{r}, \vec{p}, t)$, it is possible to approximately calculate the two-particle correlation function for small relative momenta, $\vec{q}=\frac{1}{2}\left(\vec{p}_{1}-\vec{p}_{2}\right)$, between the two emitted particles with momenta $\vec{p}_{1}$ and $\vec{p}_{2}$. Here the interaction between the two particles has to be taken into account explicitly.

To derive an expression for the two-particle correlation function, $C(\vec{P}, \vec{q})$, we assume that the finalstate interaction between the two detected particles dominates, that final-state interactions with the emitting source and all remaining particles can be neglected, that the correlation functions are determined by the two-body density of states as corrected by the interactions between the two particles, and that the single particle phase space distribution function of emitted particles, $g(\vec{p}, x)$ varies slowly as a function of momentum $\vec{p}$ (i.e. $g(\vec{p}, x) \approx g(\vec{p} \pm \vec{q}, x))$. Then the theoretical expression for the two-particle correlation function can be written as (Koonin, 1977; Pratt, 1986; Gong et al., 1991; Danielewicz and Schuck, 1992)

$$
\begin{aligned}
C(\vec{P}, \vec{q}) & =R(\vec{P}, \vec{q})+1=\frac{\Pi_{12}\left(\vec{p}_{1}, \vec{p}_{2}\right)}{\Pi_{1}\left(\vec{p}_{1}\right) \Pi_{1}\left(\vec{p}_{2}\right)} \\
& =\frac{\int d^{4} x_{1} d^{4} x_{2} g\left(\frac{1}{2} \vec{P}, x_{1}\right) g\left(\frac{1}{2} \vec{P}, x_{2}\right)\left|\phi\left(\vec{q}, \vec{r}_{1}-\vec{r}_{2}+\frac{\vec{P}\left(t_{2}-t_{1}\right)}{2 m}\right)\right|^{2}}{\int d^{4} x_{1} g\left(\frac{1}{2} \vec{P}, x_{1}\right) \int d^{4} x_{2} g\left(\frac{1}{2} \vec{P}, x_{2}\right)},
\end{aligned}
$$

where $\vec{P}=\vec{p}_{1}+\vec{p}_{2}$ is the total momentum of the particle pair. $x_{1}$ and $x_{2}$ are the space-time points of the emission of protons 1 and $2 . \Pi_{1}$ is the single- and $\Pi_{12}$ is the two-particle emission probability.

$\phi(\vec{q}, \vec{r})$ is the relative wave function of the particle pair. The effect that gives rise to the HBT effect is the identical particle interference. In the absence of any other interaction the square of the two particle wave function is then simply given by

$$
|\phi(\vec{q}, \vec{r})|^{2}=1 \pm \cos (2 \vec{q} \vec{r})
$$

where the upper sign stands for bosons and the lower for fermions.

In the presence of other interactions this result is modified. The relative wave function for Coulomb scattering is

$$
\phi_{c}(\vec{q}, \vec{r})=\exp \left(-\frac{1}{2} \pi \eta\right) \Gamma(1+i \eta) \exp (i q z){ }_{1} F_{1}(-i \eta|1| i q(r-z)),
$$

where ${ }_{1} F_{1}$ is the confluent hypergeometric series,

$$
{ }_{1} F_{1}(a|b| z)=\sum_{k=0}^{\infty} \frac{\Gamma(a+k) \Gamma(b) z^{k}}{\Gamma(a) \Gamma(b+k) k !},
$$

and $\eta=\alpha Z_{1} Z_{2} m_{r} / q$. The two-pion $\left(\pi^{+} \pi^{+}\right.$or $\left.\pi^{-} \pi^{-}\right)$relative wave function at small relative momentum is usually approximated as the symmetrized Coulomb scattering wave function

$$
\left|\phi_{\pi \pi}(\vec{q}, \vec{r})\right|^{2}=\frac{1}{2}\left|\phi_{c}(\vec{q}, \vec{r})+\phi_{c}(\vec{q},-\vec{r})\right|^{2}
$$

Due to symmetries, the wave function only depends on three independent variables which we choose to be $q, r$, and $\cos \theta=\vec{q} \cdot \vec{r} / q r$. The square of the two-pion relative wave function is displayed in fig. 
Fig. 3: Absolute square of the two-pion $\left(\pi^{+} \pi^{+}\right.$or $\left.\pi^{-} \pi^{-}\right)$relative wave function, $\left|\phi_{\pi \pi}(q, r, \cos \theta=0.5)\right|^{2}($ From Bauer $(1992 \mathrm{~b}))$.

3 as a function of $q$ and $r$ for $\cos \theta=0.5$. For $r \rightarrow 0,\left|\phi_{\pi \pi}\right|^{2}$ is given by the the Gamov penetration factor

$$
\left|\phi_{\pi \pi}(\vec{q}, 0)\right|^{2}=2 \frac{2 \pi \eta}{\exp (2 \pi \eta)-1} .
$$

For pion pairs of opposite charge $\left(\pi^{+} \pi^{-}\right)$, the wave function does not have to be symmetrized, and it is given by the hydrogen-like Coulomb wave function with $\left.\eta_{(} \pi^{+} \pi^{-}\right)=-\alpha e^{2} m_{r} / q$.

For the two-proton relative wave function the strong interaction with the prominent ${ }^{2} \mathrm{He}$-'resonance' cannot be neglected. To obtain the relative wave function in this case, we solve a radial Schrödinger equation with the Coulomb and the modified Reid soft core potential. The two-proton relative wave function is shown in fig. 4 . One can observe the peak at $q \approx 20 \mathrm{MeV} / \mathrm{c}$ due to the ${ }^{2} \mathrm{He}$-'resonance'. Due to the effect of antisymmetrization and due to the Coulomb interaction, $\left|\phi_{p p}\right| \rightarrow 0$ as $r \rightarrow 0$.

Equation 7 requires only the two-particle relative wave function and the single-particle phase space distribution function. Under the assumptions stated above it is thus possible to generate two-particle correlation functions for small relative momenta from a theory which only predicts one-particle distribution functions.

The resulting two-particle correlation function contains information on the space-time extension of the emitting source. To see this, we rewrite Equation 7 as

$$
C(\vec{P}, \vec{q})=\int d^{3} r F_{\vec{P}}(\vec{r})|\phi(\vec{q}, \vec{r})|^{2}
$$


Fig. 4: Absolute square of the two-proton relative wave function, $\left|\phi_{p p}(q, r, \cos \theta=0.5)\right|^{2}$. (From Bauer (1992b))

Here $\vec{r}=\vec{r}_{1}-\vec{r}_{2}$ is the relative coordinate of the two emitted particles, and the function $F_{\vec{P}}(\vec{r})$ is defined as

$$
F_{\vec{P}}(\vec{r})=\frac{\int d^{3} R f\left(\frac{1}{2} \vec{P}, \vec{R}+\frac{1}{2} \vec{r}, t_{>}\right) f\left(\frac{1}{2} \vec{P}, \vec{R}-\frac{1}{2} \vec{r}, t_{>}\right)}{\left(\int d^{3} r f\left(\frac{1}{2} \vec{P}, \vec{r}, t_{>}\right)\right)^{2}}
$$

where $\vec{R}=\frac{1}{2}\left(\vec{r}_{1}+\vec{r}_{2}\right)$ is the center-of-mass coordinate of the two particles, and the Wigner function $f\left(\vec{p}, \vec{r}, t_{>}\right)$is the phase space distribution of particles with momentum $\vec{p}$ and position $\vec{r}$ at some time $t_{>}$after both particles have been emitted:

$$
f\left(\vec{p}, \vec{r}, t_{>}\right)=\int_{-\infty}^{t_{>}} d t g\left(\vec{p}, \vec{r}-\vec{p}\left(t_{>}-t\right) / m, t\right) .
$$

For a given momentum $\vec{P}$, the correlation function has three degrees of freedom, $\vec{q}$, which are a function of $F_{\vec{P}}(\vec{r})$. Therefore correlation function measurements should allow the extraction of $F_{\vec{P}}(\vec{r})$, the normalized probability of two protons with the same momentum $\vec{P} / 2$ being separated by $\vec{r}$. In this sense we are in principle able to extract information on the coordinate space extension of the emitting source at the emission time of the protons.

One may also use correlation function measurements to test various theoretical models capable of predicting $g(\vec{p}, \vec{r}, t)$ and thus making specific predictions about the correlation functions. This approach is more realistic in its goals, because a full six-dimensional determination of $C(\vec{P}, \vec{q})$ is very difficult in practice. 
Fig. 5: Two-proton correlation functions calculated with a source parameterization according to Equation 16. The solid lines represent the complete calculations including the effects of quantum statistics and of the Coulomb and strong interaction. The dashed lines represent the case of only Coulomb interaction, and the dotted line is for Coulomb interaction plus the effect of the Fermi-Dirac statistics for the two protons. (From Gong et al. (1991))

\section{SENSITIVITY OF THE TWO-PROTON CORRELATION FUNCTION}

It is instructive to examine the sensitivity of the two-particle correlation function to different components of the two-particle interaction. We perform such a study for the two-proton correlation function. To do this, we use a simple zero-lifetime Gaussian source parameterization

$$
g_{0}(\vec{p}, \vec{r}, t)=\rho_{0} \exp \left(-r^{2} / r_{0}^{2}\right) \delta\left(t-t_{0}\right)
$$

where $r_{0}$ is the radius of the source. Figure 5 illustrates the effects of the different contributions to the two-proton final state interaction for sources of different radii. The Coulomb interaction dominates the shape of the correlation function for very large source radii. For $r_{0}<20 \mathrm{fm}$, the correlation function becomes increasingly sensitive to the effects of antisymmetrization and the strong interaction. The strong interaction has the dominant effect for source radii around $r_{0}=2.5 \mathrm{fm}$ due to the prominent ${ }^{2} \mathrm{He}$ resonance.

It should be pointed out at this point, however, that in a realistic calculation the use of a simple zero life-time Gaussian source parametrization is not sufficient. Instead, calculations containing the full time and momentum dependence of the emitting source are needed. 
Fig. 6: Two-proton correlation function for the reaction ${ }^{14} \mathrm{~N}+{ }^{27} \mathrm{Al}$ at $\mathrm{E} / \mathrm{A}=75 \mathrm{MeV}$, as predicted by calculations based on the BUU theory (lines) and as experimentally measured (plot symbols). (From Gong et al. (1991))

\section{CALCULATION OF TWO-PROTON CORRELATION FUNCTIONS}

We perform calculations of the single particle phase space distribution function $f(\vec{r}, \vec{p}, t)$ by numerically solving Equation 2. These single particle distributions are then inserted into Equation 7 to generate the two-particle correlation function at small relative momentum.

In Figure 6, we compare our calculations of the two-proton correlation function to experimental data for the system ${ }^{14} \mathrm{~N}+{ }^{27} \mathrm{Al}$ at a beam energy of $\mathrm{E} / \mathrm{A}=75 \mathrm{MeV}$. Since the experimental data were not triggered on impact parameter, we have to also integrate our calculations over impact parameter with the proper weighting factors. (Details of this impact parameter averaging can be found in the Appendix of Gong et al. (1991).) The experimental and theoretical correlation functions are shown as a function of the relative momentum $q$ for three different gates on the total pair momentum $|\vec{P}|=\left|\vec{p}_{1}+\vec{p}_{2}\right|$. For comparison, the beam momentum per nucleon is $p_{\text {beam }} \approx 375 \mathrm{MeV} / \mathrm{c}$.

We show two different calculations. The solid line represents the full BUU calculations with the full nucleon-nucleon cross sections. The dotted line is the result of the BUU calculation with a reduced in-medium cross section, $\sigma=\frac{1}{2} \sigma_{n n}$, where $\sigma_{n n}$ is the free space elementary nucleon-nucleon cross section. In both cases the medium corrections due to the Pauli-principle for the final nucleon scattering states are, of course, taken into account.

It is clear from this figure that sizeable differences between the two calculations with different as- 
Fig. 7: Sensitivity of the two-proton correlation function to the inmedium nucleon-nucleon cross section, $\sigma$, and to the nuclear compressibility. (After Gong et al. (1990)).

sumptions on the in-medium nucleon-nucleon cross section exist. In fact, variations of the cross section by only $10 \%$ result in differences between the calculated correlation functions which should be experimentally measurable. From this one has to conclude that two-particle correlation functions are very sensitive probes for the collisional dynamics of intermediate energy heavy ion collisions.

In fig. 7 we display the sensitivity of the two-proton correlation function to the in-medium nucleonnucleon cross section, $\sigma$, and to the value of the nuclear matter compressibility, $\kappa$. We show the results of our calculations for a stiff nuclear equation of state $(\kappa=380 \mathrm{MeV})$ and different values of the in-medium nucleon-nucleon cross section.

From our theoretical results and comparisons to experimental data we conclude that the value of the in-medium cross section (aside from correction due to the final state 'Pauli blocking') is very close to the experimentally measured (energy dependent) free value. This is in agreement with information we extracted from our investigation of the disappearance of nuclear collective flow (Krofcheck et al., 1989, 1992; Ogilvie et al., 1990; Bauer 1992a).

We also varied the compressibility of nuclear matter, which enters the calculations through the density dependence of the mean field potential $U$. In fig. 7, we also show a calculation for a soft equation of state $(\kappa=200 \mathrm{MeV})$. Here we find, however, only a weak sensitivity of our results on this parameter. This is expected, because for the relatively light system and low beam energy considered here only moderate maximum values of the nuclear density are achieved. 
Fig. 8: Comparison of theoretical calculations (histogram, Li and Bauer, 1991a) and experimental data (circles Odyniec et al., 1988) for single pion kinetic energy spectra from the reaction La+La at $E / A=1.35 \mathrm{GeV}$.

\section{CALCULATION OF TWO-PION CORRELATION FUNCTIONS}

Pluta et al. (1992) have observed that when one computes the ratio

$$
R_{\pi^{+} \pi^{-}}=\frac{\sigma_{2}\left(\pi^{+}, \pi^{-}\right)}{\sigma_{1}\left(\pi^{+}\right) \sigma_{1}\left(\pi^{-}\right)}
$$

as a function of the invariant mass of the pion pair for $1.6 \mathrm{GeV}$ protons incident on heavy target nuclei, there is a striking enhancement close to $M_{\text {inv }}\left(\pi^{+}, \pi^{-}\right)=2 M_{\pi}$.

We have investigated the possibility that the enhancement of the correlation function close to an invariant mass of $2 m_{\pi}$ could be caused by the effect of focussing of the two correlated pions into the same hemisphere due to the presence of the target spectator matter, which causes some of the produced pions to be rescattered and absorbed. To do this, we performed transport calculations with an extended BUU code, which had previously been successfully used to calculate pion spectra in heavy ion collisions ( $\mathrm{Li}$ et al. (1991)), and which has been used to show that the observed pion collective flow (Gosset et al. (1991)) was caused by nuclear shadowing effects (Li et al. (1991b)).

In this extended BUU code we propagate protons, neutrons, Delta and $\mathrm{N}^{\star}$ resonances, and pions. For their interaction cross sections, we use the experimentally measured free hadron-hadron cross sections where available. In the cases where the elementary cross sections are not measured we employ isospin symmetry and/or detailed balance to obtain the unknown quantities. The process of greatest interest here is the creation and reabsorption of pions, which is dominated by the two-step 
Fig. 9: Two pion $\left(\pi^{+}, \pi^{-}\right)$correlation function as a function of the invariant mass of the pion pair for $\mathrm{p}+\mathrm{C}, \mathrm{Pb}$ reaction at an incident energy of $1.6 \mathrm{GeV}$. The data are from Pluta et al. (1992) and are represented by the squares. Our calculations (Klakow et al. (1992)) are represented by the histograms.

process

$$
N+N \leftrightarrow N+\Delta \leftrightarrow N+N+\pi
$$

Figure 8 (Li and Bauer, 1991a) shows the single pion spectra calculated in this model. The reaction is $\mathrm{La}+\mathrm{La} \rightarrow \pi^{-}+\mathrm{X}$ at $E / A$ is $1.35 \mathrm{GeV}$. The data of Odyniec et al. (1988) are represented by the circles, and the results of the calculation are shown by the histogram. Experiment and theory were both gated for central collisions $(b \leq 2.8 \mathrm{fm})$, and the detection angle for the pions was $90^{\circ} \pm 30^{\circ}$ in the center of mass of target and projectile. More recent calculations (Li et al., in preparation) use improved parametrizations for the elementary cross sections and shown an even better agreement with experimental data.

To generate correlation functions which are comparable to experiment, we calculate the correlated pairs from events with fixed reaction plane

$$
M_{\mathrm{inv}}=\sqrt{\left(E_{1}+E_{2}\right)^{2}-\left(\vec{p}_{1}+\vec{p}_{2}\right)^{2}}
$$

whereas we compute the uncorrelated background events from

$$
M_{\mathrm{inv}, \phi}=\sqrt{\left(E_{1}+E_{2}\right)^{2}-\left(\vec{p}_{1}+\mathcal{R}_{\phi} \vec{p}_{2}\right)^{2}}
$$


where $E_{i}$ and $\vec{p}_{i}$ are the energy and momentum of pion $i$ in the some frame of reference (we chose the lab frame). The operator $\mathcal{R}_{\phi}$ rotates a vector $\vec{p}$ by a (random) angle $\phi$ about the beam axis (the $z$-axis in our case):

$$
\mathcal{R}_{\phi} \vec{p}=\left(\begin{array}{ccc}
\cos \phi & -\sin \phi & 0 \\
\sin \phi & \cos \phi & 0 \\
0 & 0 & 1
\end{array}\right)\left(\begin{array}{l}
p_{x} \\
p_{y} \\
p_{z}
\end{array}\right)
$$

The correlation function is then computed from

$$
R\left(M_{\mathrm{inv}}\right)=\frac{N\left(M_{\mathrm{inv}}\right)}{N\left(\left\langle M_{\mathrm{inv}, \phi}\right\rangle_{\phi}\right)}
$$

With this technique we approximate the experimental fact that for the generation of the background events one is forced to average over random relative orientations of the reaction planes for the two uncorrelated pions in every pair, whereas the correlated pairs are all sampled with fixed reaction plane of the pair and therefore contain the effects of the target matter shadowing at finite impact parameter. For a more detailed description of the numerical procedure and the effect of pion absorption on two pion correlation functions see Klakow et al. (1992).

In fig. 9, we compare the results of our calculations with the experimental data for the $\mathrm{C}$ and $\mathrm{Pb}$ targets. We find that we can reproduce the $\mathrm{Pb}$ target data by the calculations, whereas we slightly overpredict the correlation function at small $M_{\text {inv }}$ for the $\mathrm{C}$ target.

The main result of this investigation is thus that the effect of shadowing due to pion absorption has a large effect on the extracted two-pion correlation function, particularly in asymmetric projectiletarget systems. As shown this kinematic effect leads to an enhancement of the two-pion correlation function at small $M_{\mathrm{inv}}$ and can lead to wrong extracted source sizes if not included.

\section{CONCLUSIONS}

The calculation of two-particle correlation functions at small relative momentum on the basis of one-body transport theories is feasible by using the convolution techniques described above. Thus intensity interferometry is a powerful tool to test nuclear transport theories and to investigate nuclear dynamics.

Comparisons with experiment show that the BUU transport theory is able to reproduce detailed features of the experimentally measured two-proton correlation functions. These features include the pair momentum dependence of the peak due to the ${ }^{2} \mathrm{He}$-'resonance', the effect of source deformation, and the lifetime effect on the correlation function.

We have shown that the theoretically obtained two-proton correlation functions are sensitive to the value of the in-medium nucleon-nucleon cross section. At higher beam energies and for large systems we also expect a sensitivity of the results on the compressibility of nuclear matter. Thus nuclear intensity interferometry is a useful tool to investigate the nuclear transport properties, and it should also enable us to conduct further studies of the nuclear equation of state.

From a theoretical standpoint it is clearly desirable to compare to impact parameter resolved experimental data, which will further increase the sensitivity of two-particle correlation functions at small relative momentum to the effects discussed above. These studies are currently in progress. 
For the two-pion correlation functions we have shown that it is essential to included the effects of pion rescattering and absorption properly into the calculations. A source characterization without taking these effects into account will lead to wrong results for quantities like the size of the emitting source.

For the next few years, we anticipate that the focus of HBT type of studies in heavy ion collisions will also shift to the energy range of $E / A \approx 1 \mathrm{GeV}$. At this energy range we expect much higher compression $\left(\rho / \rho_{0} \approx 2-4\right)$ of the colliding nuclei than at the beam energy range considered here $\left(E / A \approx 100 \mathrm{MeV}\right.$, and $\left.\rho / \rho_{0} \approx 1-3-1.5\right)$. Then two-particle correlations should be able to give us additional information on the nuclear compressibility and thus provide valuable insight into the nuclear equation of state. Work in this direction is alrady in progress (Mader and Bauer, 1992).

\section{ACKNOWLEDGMENTS}

This work was supported by the National Science Foundation under Grants No. 89-06116 and 9017077 as well as by a Presidential Faculty Fellow award. Helpful conversations and collaborations with G.F. Bertsch, P. Danielewicz, C.K. Gelbke, W.G. Gong, D. Klakow, C. Mader, S. Pratt, and P.Schuck are gratefully acknowledged.

\section{REFERENCES}

Aichelin, J. and Bertsch, G.F. (1985). Numerical simulation of medium energy heavy ion reactions. Phys. Rev. C 31, 1730.

Aichelin, J. and Ko, C.M. (1985). Subthreshold kaon production as a probe of the nuclear equation of state. Phys. Rev. Lett. 55, 2661.

Aichelin, J. et al. (1985). Importance of momentum-dependent interactions for the extraction of the nuclear equation of state from high-energy heavy-ion collisions. Phys. Rev. Lett. 58, 1926.

Aichelin, J. and Stöcker, H. (1986). Quantum molecular dynamics - A novel approach to N-body correlations in heavy ion collisions. Phys. Lett. B176, 14.

Aichelin, J. (1991). "Quantum" molecular dynamics - a dynamical microscopic n-body approach to investigate fragment formation and the nuclear equation of state in heavy ion collisions. Phys. Rep. 202, 233.

Ardouin, D. et al. (1988). Evidence for persisting mean field effects at $\mathrm{E} / \mathrm{A}=60 \mathrm{MeV}$ from particleparticle correlation measurements and theoretical investigations with the Landau Vlasov equation. Z. Phys. A 329, 505.

Ayik, S. and Grégoire, C. (1988). Fluctuations of single-particle density in nuclear collisions. Phys. Lett. B212, 269.

Ayik, S. and Grégoire, C. (1990). Transport theory of fluctuation phenomena in nuclear collisions. Nucl. Phys. A 513, 187.

Ayik, S. et al. (1992). The Boltzmann-Langevin model for nuclear collisions. Nucl. Phys. A 545, $35 \mathrm{c}$.

Bauer, W. et al. (1986). Energetic photons from intermediate energy proton and heavy ion induced reactions. Phys. Rev. C 34, 2127.

Bauer, W. et al. (1987). Fluctuations and clustering in heavy ion collisions. Phys. Rev. Lett. 58, 863.

Bauer, W. (1987a). Light particle correlations in heavy ion reactions. Nucl. Phys. A 471, 604.

Bauer, W. (1988). Nuclear stopping at intermediate beam energies. Phys. Rev. Lett. 61, 2534. 
Bauer, W. (1989). Unified calculation of photon and pion spectra in intermediate energy heavy ion reactions. Phys. Rev. C 40, 715 (1989).

Bauer, W. et al. (1992). Hadronic interferometry in heavy ion collisions. Annu. Rev. Nucl. Part. Sci. 42, 77.

Bauer, W. (1992a). Hadronic transport properties in intermediate energy heavy ion collisions. Nucl. Phys. A538, 83c.

Bauer, W. (1992b). Nuclear dynamics and intensity interferometry. Nucl. Phys. A545, 369c.

Bauer, W. et al. (1992c). Bubble and ring formation in nuclear fragmentation. Phys. Rev. Lett. 69, 1888.

Benhassine, B. et al. (1992). Phase space fluctuations and dynamics of fluctuations of collective variables. Nucl. Phys. A 545, 81c.

Bertsch, G.F. et al. (1984). Boltzmann equation for heavy ion collisions. Phys. Rev. C 29, 673.

Bertsch, G.F. et al. (1988). Pion interferometry in ultrarelativistic heavy-ion collisions. Phys. Rev. $C$ 37, 1896.

Bertsch, G.F. and Das Gupta, S. (1988). A guide to microscopic models for intermediate energy heavy ion collisions. Phys. Rep. 160, 189.

Boal, D.H. et al. (1990). Intensity Interferometry in subatomic physics. Rev. of Mod. Phys. 62, 553.

Bonasera, A. et al. (1992). Fluctuations of the one-body distribution function. Phys. Rev. C 46, 1431.

Bonasera, A. et al. (1992a). Quasistationary description of fluctuations. Nucl. Phys. A 545, 71c.

Burgio, F. et al. (1991). Fluctuations in nuclear dynamics. From transport theory to dynamical simulation. Nucl. Phys. A 529, 157.

Burgio, F. et al. (1991). Dynamical clusterization in the presence of instabilities. Phys. Rev. Lett. 69, 885.

Cassing, W. et al. (1990). Production of energetic particles in heavy-ion collisions. Phys. Rep. 188, 363.

Cassing, W. and Mosel, U. (1990). Many-body theory of high-energy heavy-ion reactions. Prog. Part. Nucl. Phys. 25, 235.

Chitwood, C.B. et al. (1986). Light particle emission in ${ }^{16} \mathrm{O}$-induced reactions on ${ }^{12} \mathrm{C},{ }^{27} \mathrm{Al}$, and ${ }^{197} \mathrm{Au}$ at $\mathrm{E} / \mathrm{A}=25 \mathrm{MeV}$. Phys. Rev. C 34, 858.

Cugnon, J. et al. (1981). Equilibration in relativistic nuclear collisions. A Monte Carlo calculation. Nucl. Phys. A 352, 505.

Cugnon, J. et al. (1982). Pion production in central high energy nuclear collisions. Nucl. Phys. A 379, 553 .

Cugnon, J. et al. (1987). Medium effects in the nuclear Landau-Vlasov transport theory. Phys. Rev. C 35, 861.

Cugnon, J. and Lemaire, M.C. (1988). Medium effects in pion production. Nucl. Phys. A 489, 781.

Danielewicz, P. and Bertsch, G.F. (1991). Production of deuterons and pions in a transport model of energetic heavy ion reactions. Nucl. Phys. A 533, 712.

Danielewicz, P. and Schuck, P. (1992). Formulation of particle correlation and cluster production in heavy-ion induced reactions. Phys. Lett. B274, 268.

Danielewicz, P. and Pan, Q. (1992). Blast of light fragments from central heavy-ion collisions. Phys. Rev. C 46, Number 5.

Fox, D. et al. (1986). Large angle correlations in $40 \mathrm{MeV} /$ nucleon ${ }^{12} \mathrm{C}+\mathrm{C}$. Phys. Rev. C 33, 1540.

Gale, C. (1987). Pion multiplicities in dynamical models of heavy ion collisions. Phys. Rev. C 36, 2152.

Gale, C. et al. (1987). Heavy-ion collision theory with momentum-dependent interactions. Phys. Rev. C 35, 1666.

Goldhaber, G. et al. (1960). Influence of Bose-Einstein statistics on the antiproton-proton annihilation process. Phys. Rev. 120, 300. 
Gong, W.G. et al. (1990). Intensity interferometric test of nuclear collision geometries obtained from the Boltzmann-Uehling-Uhlenbeck equation. Phys. Rev. Lett. 65, 2114.

Gong, W.G. et al. (1991). Space-time evolution of nuclear reactions probed by two-proton intensity interferometry. Phys. Rev. C 43, 781.

Gong, W.G. et al. (1991a). Space time evolution of the reactions ${ }^{14} \mathrm{~N}+{ }^{27} \mathrm{Al},{ }^{197} \mathrm{Au}$, at $\mathrm{E} / \mathrm{A}=75$ $\mathrm{MeV}$ and ${ }^{129} \mathrm{Xe}+{ }^{27} \mathrm{Al},{ }^{122} \mathrm{Sn}$ at $\mathrm{E} / \mathrm{A}=31 \mathrm{MeV}$ probed by two-proton intensity interferometry. Phys. Rev. C 43, 1804.

Gosset, J. et al. (1989). Nuclear collective flow and charged pion emission in Ne-nucleus collisions at $\mathrm{E} / \mathrm{A}=800 \mathrm{MeV}$. Phys. Rev. Lett. 62, 1251.

Grégoire, C. et al. (1985). Semiclassical approaches to proton emission in intermediate-energy heavyion reactions. Nucl. Phys. A 436, 365.

Grégoire, C. et al. (1987). Semiclassical dynamics of heavy-ion reactions. Nucl. Phys. A 465, 317.

Hanbury Brown, R., and Twiss, R.Q. (1954). Phil. Mag. 45, 663.

Hanbury Brown, R., and Twiss, R.Q. (1956). Nature 177, 27.

Hanbury Brown, R., and Twiss, R.Q. (1956). A test of a new type of stellar interferometer on sirius. Nature 178, 1046.

Li, B.A. and Bauer, W. (1991). Two-temperature shape of pion spectra in relativistic heavy ion collisions. Phys. Lett. B254, 335.

Li, B.A. and Bauer, W. (1991a). Pion spectra in a hadronic transport model for relativistic heavy ion collisions. Phys. Rev. C 44, 450.

Li, B.A. et al. (1991b). Preferential emission of pions in asymmetric nucleus-nucleus collisions. Phys. Rev. C 44, 2095.

Klakow, D. (1992) Work in preparation.

Koonin, S.E. (1977). Proton pictures of high-energy nuclear collisions. Phys. Lett. B70, 43 (1977).

Krofcheck, D. et al. (1989). Disappearance of flow in heavy ion collisions. Phys. Rev. Lett. 63, 2028.

Krofcheck, D. et al. (1992). Disappearance of flow as a probe of the nuclear equation of state. Phys. Rev. C 46, 1416.

Kruse, H. et al. (1985). Microscopic theory of pion production and sidewards flow in heavy-ion collisions. Phys. Rev. Lett. 54, 289.

Kruse, H. et al. (1985a). Vlasov-Uehling-Uhlenbeck theory of medium energy heavy ion reactions: role of mean field dynamics and two body collisions. Phys. Rev. C 31, 1770.

C. Mader and W. Bauer (1992). Work in progress.

Odyniec, G. et al. (1988). In: Proceedings of the 8th High Energy Heavy Ion Study, Berkeley, edited by J. Harris and G. Wozniak, LBL Report 24580, p. 215

Ogilvie, C.A. et al. (1990). The disappearance of flow and its relevance to nuclear matter physics. Phys. Rev. C 42, R10.

Ono, A. et al. (1992). Prog. of Theor. Phys. 87, 1185.

Peilert, G. et al. (1992). Dynamical treatment of Fermi motion in a microscopic description of heavy ion reactions. Phys. Rev. C 46, 1457.

Pluta, J. et al. (1992). Work in preparation.

Pratt, S. (1984). Pion interferometry for exploding sources. Phys. Rev. Lett. 53, 1219.

Pratt, S. (1986). Pion interferometry of the quark-gluon plasma. Phys. Rev. D 33, 1314.

Randrup, J. and Remaud, B. (1990). Fluctuations in one-body dynamics. Nucl. Phys. A 514, 339.

Randrup, J. (1992). Fluctuations and correlations in nuclear one-body dynamics. Nucl. Phys. A 545, 47c.

Reinhard, P.-G. and Suraud, E. (1992). Stochastic TDHF and large fluctuations. Nucl. Phys. A $\mathbf{5 4 5}, 59 \mathrm{c}$.

Schuck, P. et al. (1989). Semiclassical and phase space approaches to dynamical and collisional problems of nuclei. Prog. Part. Nucl. Phys. 22, 181.

Shuryak, E.V. (1973). The correlations of identical pions in multibody production. Phys. Lett. B44, 
387.

Stöcker, H. and Greiner, W. (1986). High energy heavy ion collisions - probing the equation of state of highly excited hadronic matter. Phys. Rep. 137, 277.

Stock, R. et al. (1982). Compression effects in relativistic nucleus-nucleus collisions. Phys. Rev. Lett. 49, 1236.

Wang, S.J. et al.(1991). Relativistic transport theory for hadronic matter. Ann. Phys. (N.Y.) 209, 251.

Wong, C.Y. (1982). Dynamics of nuclear fluid. VIII. Time-dependent Hartree-Fock approximation from a classical point of view. Phys. Rev. C 25, 1460.

Zhu, F. et al. (1991). Light-particle correlations and the ${ }^{3} \mathrm{He}+\mathrm{Ag}$ reaction at $200 \mathrm{MeV}$. Phys. Rev. C 44, R582. 